\section{Eloísa Díaz Inzunza: entre la medicina, la psicología y la educación}

\author{
GONZALO SALAS ${ }^{1, \mathrm{a}, \mathrm{b}}$, HERNÁN SCHOLTEN ${ }^{2, \mathrm{~b}}$, \\ LUISA GUERRA-LABBÉ ${ }^{3, c}$, JOSÉ RAMOS-VERA ${ }^{1, d}$, \\ ELIZABETH PARDO-GONZÁLEZ ${ }^{1, \mathrm{e}}$
}

\section{Eloísa Díaz: between medicine, psychology and education}

Eloisa Díaz Insunza, the first Latin American female physician, completed her studies in Medicine and Surgery at the University of Chile in 1887 and worked a large part of her life as Medical Inspector of Public Schools of Santiago, Chile. In this article, the focus is placed on her "Test Memorandum" (1886) and her first Reports to the Ministry of Public Education (1899-1905), to appreciate the hygienist perspective that characterizes her proposals. We describe her intervention project that sought to integrate medical practice with psychology and education, to solve a social problem such as the degeneration of the Chilean race.

(Rev Med Chile 2019; 147: 499-504)

Key words: Education; History of Medicine; Hygiene; Psychology; Women.
'Universidad Católica del Maule. Talca,

Chile.

${ }^{2}$ Universidad de Buenos Aires. Buenos

Aires, Argentina.

${ }^{3}$ Universidad Autónoma de Chile. Chile.

a Doctor en Educación.

bsicólogo.

'Terapeuta Ocupacional

dEstudiante de Doctorado en Psicología

eEstudiante de Magíster en Salud

Mental Infanto-Juvenil.

Fondos: Este artículo fue apoyado por una subvención de la Comisión Nacional de Investigación Científica y Tecnológica (CONICYT), a través del Fondo Nacional de Desarrollo Científico y Tecnológico. Proyecto Fondecyt 11150436

Los autores declaran no tener conflictos de interés.

Recibido el 14 de noviembre de 2018, aceptado el 15 de marzo de 2019.

Correspondencia a:

Gonzalo Salas

Departamento de Psicología, Facultad de Ciencias de la Salud. Universidad Católica del Maule, Talca, Chile. gsalas@ucm.cl
$\mathrm{H}$ acia finales del siglo XIX, las mujeres latinoamericanas lograban que se reconociera su derecho a la educación superior. En ese contexto, se graduaban las primeras médicas formadas en universidades locales: Matilde Montoya (México) y Rita Lobato (Brasil) obtuvieron sus respectivos títulos en agosto y diciembre de 1887; dos años más tarde, Cecilia Grierson se transformó en la primera médica argentina y, para 1900, Laura Esther Rodríguez Dulanto egresaba de la Universidad Nacional Mayor de San Marcos de Lima (Perú) ${ }^{1-4}$. Un poco más tardíos son los casos de Colombia y Venezuela: Inés Ochoa de Patiño fue la primera mujer graduada como médica en Colombia, en 1945, y la venezolana Sara Bendahan terminó sus estudios en 1930, pero su título le fue entregado casi diez años después por problemas políticos ${ }^{5}$.

Sin embargo, la primera médica graduada en América Latina fue Eloísa Díaz Insunza (1866-
1950). Nacida en el marco de una familia económicamente acomodada de Santiago de Chile, ingresó en las aulas de la academia en 1881, tras conseguir el grado de bachiller en humanidades en el Instituto Nacional. Obtuvo su licenciatura en Medicina y Farmacia en diciembre de 1886, y su título en Medicina y Cirugía en enero de 1887, diez años después de que las mujeres chilenas conquistaran el acceso a las aulas universitarias ${ }^{6-10}$.

Su memoria de prueba y sus primeros pasos en la práctica médica la mostraron especialmente interesada en la ginecología, área a la que se abocó entre 1888 y 1891. En paralelo, trabajó en la Escuela Normal de Preceptores del Sur como médica y profesora de Higiene, cargos que mantuvo hasta 1897, cuando fue nombrada Médico Inspector de las Escuelas Públicas de Santiago, tarea a la que dedicó la mayor parte de su vida.

Este artículo propone un recorrido por algunos hitos de su biografía, que se inician con su 
memoria de prueba para obtener su licenciatura en Medicina y Farmacia y se enfoca luego en su labor como médico inspector. De este modo, se busca poner de relieve la particular modalidad en que llevó adelante su práctica profesional y abordó problemáticas que conectan la medicina con la psicología y la educación. Para ello, es necesario detenerse brevemente en las características del higienismo o "ciencia de la higiene", para mostrar que ese fue el enfoque desde el cual formuló propuestas que, si bien concernían principalmente a las mujeres y a la niñez, impulsaban transformaciones en el conjunto de la sociedad chilena.

\section{Higienismo y medicina}

Aunque hay referencias a la cuestión de la higiene en la medicina antigua y medieval, fue a finales del siglo XVIII que adquirió una relevancia notable en el ámbito médico ${ }^{11}$. En efecto, a partir de los aportes de Lavoisier y de Pasteur, la prevención se convertirá en una cuestión de primer orden para la medicina, al mismo tiempo que la figura del médico asumirá una creciente importancia y se impondrá una nueva agenda: ya no se trata simplemente de atender a los enfermos sino, preferentemente, de evitar la aparición y propagación de la enfermedad. La invención y amplia difusión de la vacuna durante el siglo XIX, es un ejemplo ilustrativo en este sentido. Esta preocupación por la profilaxis impulsará vastas reformas sociales, políticas y culturales que, desde el Estado y sus organismos, buscaron intervenir, modificar $y$, eventualmente, exterminar todo aquello que pudiera poner en peligro la salud de la población. De estas cuestiones se ocupó ampliamente el filósofo-historiador Michel Foucault, cuyos análisis permiten apreciar claramente la articulación de diversos saberes y prácticas (entre los que se destaca la medicina) con los particulares mecanismos de poder que comienzan a desplegarse en la Europa decimonónica ${ }^{12,13}$.

En el caso de Chile, es posible observar una difusión inicial del higienismo a mediados del siglo XIX con la aparición del primer manual de higiene en $1859^{14}$, el cual logró imponerse tras algunas polémicas iniciales sobre el rol del Estado en la protección de la salud de la población, dada la resistencia de legisladores e intelectuales liberales. Adolfo Murillo fue uno de los primeros médicos en difundir el paradigma higienista a nivel local. En 1872, luego de que presentara un informe sobre la educación física y la higiene al gobierno chileno, se aprobó el decreto de enseñanza de la higiene en las escuelas. Además, en 1877, se impulsó un primer proyecto de ley de vacunación obligatoria y, unos años más tarde, se establecerá la Junta Central de la Vacuna. En 1884, Ricardo Dávila Boza, considerado un pionero de la infectología en Chile, publicó "La hijiene de la escuela", en donde propuso, por primera vez, incluir el ámbito escolar en la agenda sanitaria local. En 1892, como producto de la preocupación por varios brotes epidémicos, se crearon el Consejo Superior de Higiene Pública y el Instituto de Higiene ${ }^{15}$. La creación del puesto de inspector sanitario, en 1898, y la sanción del primer Código Sanitario, en 1918, permiten dar cuenta de algunos hitos en el avance de las ideas higienistas en Chile hasta comienzos del siglo XX.

\section{Las patologías femeninas desde una perspectiva higienista}

Este pequeño desvío por la cuestión del higienismo y las breves referencias a su difusión en Chile, permitirán contextualizar la producción de Eloísa Díaz y mostrar que su enfoque fue, al interior del panorama local, sumamente particular y novedoso.

Esto resulta apreciable tempranamente en su producción, en la ya mencionada memoria de prueba de 1886. En efecto, sus "Breves observaciones sobre la aparición de la pubertad en la mujer chilena y las predisposiciones patológicas del sexo"16 exceden ampliamente las cuestiones ginecológicas que el título parece sugerir. La propia autora caracteriza su tema de investigación como una "preocupación social" y afirma que busca contribuir al estudio de las afecciones que impactan sobre la población local, a la luz de sus "condiciones de vida, clima i costumbres”. Y es por eso que, apoyándose esencialmente en fuentes francesas, se presenta allí el mecanismo fisiológico de la menstruación como parte de una serie de cambios determinados por el sistema nervioso, cuyo impacto no se limita al ovario, sino que influyen también en su "ser moral", y que, con frecuencia, puede derivar en el histerismo o en ataques epileptiformes. De este modo, incorpora en sus análisis algunos tópicos de la psicopatología femenina que se discutían en esa 
época, especialmente a partir de las experiencias del neurólogo francés Jean-Martin Charcot en La Salpetrière ${ }^{17}$.

En la segunda parte de su memoria, presenta un estudio que busca dar cuenta del momento de aparición de la menstruación en la mujer chilena, basándose en datos estadísticos de 4.600 sujetos. En esta sección tampoco se limita a presentar datos fisiológicos, sino que incorpora los aspectos sociológicos, geográficos e incluso meteorológicos de las tres grandes regiones en las que la autora divide el territorio chileno (norte, centro o agrícola y austral o insular). Por ejemplo, además de adjudicarle un papel determinante al clima y a la alimentación en la prematura aparición de la menstruación de la mujer norteña, también asigna un rol significativo a su vida activa. Mientras que, en la región central, se haría notar "el influjo poderoso que (...) ejerce la cultura i la vida del gran mundo sobre el desarrollo corporal $i$ sobre la aparición de la menstruación"16.

A este análisis, que integra elementos heterogéneos, añade una clasificación y estudio de las mujeres chilenas que pone de relieve las diferencias entre tres grupos diferentes: la mujer de las clases acomodadas, las campesinas y las trabajadoras urbanas. Su foco de preocupación se concentra en este último caso, por las "notables perturbaciones" que sufriría el organismo de estas mujeres por su "vida de quejumbres $i$ miserias", lo cual llevaría a que "surja el crimen, como una esperanza i el vicio i corrupcion como un lenitivo de semejantes sufrimientos" 16 . Y en tanto se trata del grupo de mujeres con mayor fecundidad, la autora estima necesario resolver esta situación a través de los medios que indiquen los hombres de ciencia para evitar "la decadencia inminente de nuestra raza"16. Esta última afirmación introduce la temática de la raza, que será retomada más adelante, y permite apreciar la difusión en Chile de la teoría de la degeneración que, de acuerdo a la propuesta del psiquiatra francés Bénédict Morel (1809-1873), planteaba la transmisión a la descendencia de los rasgos patológicos de los progenitores ${ }^{18}$. En este sentido, la médica chilena recomienda, como medidas iniciales, que el Estado construya viviendas para obreros, aumente los salarios y vigile las tabernas, expresando ya en este temprano texto su preocupación por los peligros del alcoholismo, que retomará en varios trabajos posteriores.

Esta breve presentación de su memoria de prueba busca mostrar que, aunque se trata de un estudio enfocado en la mujer (desde una perspectiva que, como fue señalado, incluye aspectos fisiológicos, psicológicos e incluso sociológicos), deriva finalmente en un abordaje claramente profiláctico. A continuación, podrá apreciarse que esa perspectiva será retomada e incluso profundizada en producciones posteriores.

\section{"La higiene en la escuela es precursora de la higiene en el hogar": su labor como Médico Inspector}

Las posteriores publicaciones de Eloísa Díaz se reducen esencialmente a los informes que redactó al Ministerio de Instrucción Pública y las ponencias que presentó en los congresos médicos latino-americanos (Santiago, 1901; Buenos Aires, 1904) a partir de su nombramiento como Médico Inspector de las Escuelas Públicas de Santiago en $1898^{19}$.

En estos textos se advierte con mayor amplitud y precisión la perspectiva higienista que subtiende sus análisis y propuestas, que ahora apuntaban al niño escolar proveniente de las clases populares de Santiago. Interesa aquí señalar los principales problemas abordados allí por la autora, a veces de manera insistente.

1. En primer lugar, las condiciones edilicias de las escuelas, temática casi omnipresente en sus textos y que introduce varios tópicos comunes de la literatura higienista. Por ejemplo, las insistentes solicitudes por una adecuada iluminación y ventilación de las aulas, la construcción de letrinas, saliveros y la reparación de las acequias, como medios de impedir la proliferación de epidemias. Esta preocupación se extendía a las caballerizas, casas de tolerancia y especialmente a las licorerías ubicadas en cercanías de las escuelas. Más que la cuestión toxicológica, a la médica chilena le preocupa "el espectáculo de acciones impropias que pugnan contra la moral y que pervierten los sentimientos del niño"20.

2. En sus informes, se ocupa también de destacar la importancia de la gimnástica, argumentando que "en todos los países civilizados la educación física marcha unida a la intelectual y moral"19. En este sentido, sus textos se detienen con cierto detalle en las ventajas fisiológicas del ejercicio muscular 
moderado y promueven la creación o acondicionamiento de los espacios dedicados a la gimnasia en el ámbito escolar.

3. La articulación entre educación y moral, la lleva a introducir tópicos propios de la literatura psicológica en esa época, como la cuestión de las pasiones, los hábitos, el carácter, etc. Por ejemplo, en el informe presentado el 5 de junio de 1901, sostiene que "la influencia que la escuela ejerce sobre el modo de ser de los niños" ya que "en ella aprende el buen trato y la sociabilidad"20. Ese mismo año, en el Primer Congreso Latino-Americano de Medicina, se ocupó más ampliamente de esta cuestión y sostuvo que en las escuelas primarias "se adquieren los buenos i malos hábitos, donde se forman las nobles o perversas inclinaciones, las ideas exactas o falsas", por lo cual allí "debe enseñársele al niño todos los medios necesarios para la conservación de su salud"19.

De modo todavía más explícito se muestra este vínculo entre la educación, los aspectos anímicos y la moral cuando compara el papel de la higiene con el de la religión:

La relijión y la higiene enseñan al hombre el completo dominio sobre sus pasiones i malos hábitos $i$ extienden su benéfica influencia en el hogar mismo, enseñando el orden i reglamentando todos los preceptos para vivir largos años en perfecta paz i completa salud ${ }^{20}$.

Finalmente, amerita al menos ser mencionada su propuesta de establecer la obligatoriedad de los paseos escolares, en virtud de sus ventajosos aspectos morales ${ }^{18}$. En efecto, el impacto que la belleza del paisaje imprimiría en el alma del niño, lo llevaría a adquirir "el irresistible hábito de repetir estas nobles impresiones" y lo motivará a buscar distracciones honestas y a alejarse del vicio, al mismo tiempo que incrementaría "su amor a la tierra nativa"20. Para fundamentar esta propuesta, la médica chilena no se apoya en literatura médica, sino en las propuestas del pedagogo alemán Friedrich Froebel, en su célebre texto de 1826 titulado "La educación del hombre"21.

En resumen, la articulación entre educación y moral que propone Eloísa Díaz, permite apreciar una concepción de salud que remite no solo al organismo, sino también a la dimensión anímica e, incluso, al ámbito familiar. Y, a su vez, la es- cuela es presentada como un espacio que articula cuestiones médicas y pedagógicas con el desarrollo intelectual y el carácter de los niños. Es posible afirmar que, en este sentido, la escuela es concebida por la médica chilena como un agente civilizador ${ }^{22}$.

4. Desde un enfoque higienista, ya estaba presente en su memoria de 1886, la temática de la raza chilena. En efecto, es posible afirmar que sus propuestas expuestas hasta aquí deben ser consideradas a la luz de su preocupación respecto de la situación racial en Chile, cuya gravedad ameritaría una inmediata intervención de las autoridades.

Ya en 1899, planteaba que era notorio a simple vista el decaimiento de las energías físicas e intelectuales en casi todos los escolares, lo cual tendría funestas consecuencias sobre los niños, ya que era la principal fuente de perturbaciones nerviosas. Este fenómeno, a su juicio, sería "el principal factor del prematuro decaimiento que ya se nota en nuestra raza"20. De este modo, enlazando los aspectos orgánicos y anímicos, la autora presenta un cuadro de la población infantil que plantea un horizonte eventualmente catastrófico para la sociedad chilena. Es por ello que define su interés y su deber como funcionario público, en tanto médico inspector, con los siguientes términos: “...proporcionar (...) a los niños de nuestras escuelas todo lo necesario para que (...) puedan más tarde ser ciudadanos útiles a su patria y den siempre pruebas del vigor y la energía de nuestra raza, que desgraciadamente hoy día está tan degenerada”20.

En este sentido, como lo señala la frase que fue escogida como título de este apartado, Eloísa Díaz se propuso convertir a la escuela en un espacio sanitario cuya efectividad trasciende el caso individual y se proyecta hacia lo social, en primera instancia hacia el espacio familiar: "la higiene en la escuela es precursora de la higiene en el hogar"19,20.

\section{Conclusión}

Como fue anunciado previamente, a lo largo de este trabajo se enfatizó en un aspecto algo descuidado en los estudios sobre la trayectoria de Eloísa Díaz: la perspectiva higienista presente tanto en su temprana memoria de prueba (1886) como en sus primeros informes como médico inspector (1899$1905)^{16,19}$. Esta perspectiva continuará presente cuando asuma la dirección del Servicio Médico 
Escolar de Chile en 1911, cargo que ocupará hasta su retiro en 1925.

En efecto, más allá del cambio de temática desde sus estudios iniciales sobre las patologías femeninas hasta su posterior interés por el ámbito escolar, hay una continuidad bastante clara en su producción: la salud de la población y el porvenir de la propia raza chilena. En este sentido, los análisis que realizaba y las diversas intervenciones que proponía excedían el marco de la clínica médica e introducían la consideración de factores psicológicos e incluso sociológicos. Como lo muestran con mayor claridad sus textos sobre la situación de las escuelas chilenas, para lograr una efectiva profilaxis era preciso intervenir no solamente sobre los cuerpos, sino también sobre el alma (sobre los comportamientos, las pasiones, los hábitos, el carácter) e, incluso, sobre aspectos ambientales que, además de las condiciones edilicias, remitían al entorno familiar. La escuela permitía integrar, con mayor o menor facilidad, todos estos elementos en el marco de un espacio que, paulatinamente, comenzaba a asumir una considerable complejidad y cuya función desborda ampliamente lo educativo ${ }^{22}$.

De este modo, a través de sus inspecciones e informes, buscó sensibilizar a sus colegas y a las autoridades sobre la situación de la población escolar chilena. Participó en la configuración de un objeto de intervención que, en adelante, se ampliará y profundizará cada vez más: la infancia. Y permitirá comenzar a comprender que, una intervención eficaz y exitosa en esos sujetos, exigía el concurso y la intervención de saberes diversos, provenientes de áreas como la psicología, la educación e incluso la sociología.

De este modo, se asoman otros aspectos de su producción que resultan sumamente atractivos para las investigaciones históricas, como es el caso de la concepción de la infancia presente en sus textos, que ubican al niño como una víctima y mismo tiempo como una inversión ${ }^{23}$. Eloísa Díaz llegó a promover, en 1899, la creación de una sociedad protectora de la infancia ${ }^{20}$ que, además de recibir fondos públicos, contaría con la colaboración de los médicos y el aporte de la caridad a través de las damas de sociedad. Se anticipaba así a la creación, dos años más tarde, del Patronato Nacional de la Infancia, que continúa funcionando en la actualidad como Patronato Madre-Hijo. Esta misma perspectiva estuvo presente, en esos años, en la legislación sobre la infancia desvalida y en el Primer Congreso Nacional de Protección a la Infancia (1912). En este sentido, el abordaje de su producción engloba también un capítulo en la historia de la infancia en Chile ${ }^{24}$.

Por otra parte, queda todavía pendiente indagar su relevancia en el marco de una historia de la pediatría en Chile que, tras vacilar entre varias denominaciones (puericultura, nipiología, pedología, etc.), se consolidará durante la tercera década del siglo XX y abrirá el camino a posteriores intervenciones de los profesionales médicos, como la higiene mental y la psiquiatría infantil ${ }^{25}$.

\section{Referencias}

1. Montoya M. Fuentes para el análisis de la educación de la mujer mexicana finisecular. Signos Históricos 2016; 18 (36): 182-192.

2. Lobo FB. Rita Lobato: a primeira médica formada no Brasil. Revista de História 1971; 42 (86): 483-5.

3. Pérgola F. Cecilia Grierson, primera médica argentina. Rev Argent Salud Pública 2015; 6 (24): 47-8.

4. Díaz Núñez H. Primera médica peruana, Dra. Laura Esther Rodríguez Dulanto (1872-1919). Anales de la Facultad de Medicina 2007, 68 (2): 181-4.

5. Cohen LM. Colombianas a la Vanguardia. Medellín: Editorial Universidad de Antioquia, 2001.

6. Jiménez EA. Dra. Eloísa Díaz Insunza. Rev Chilena Infectol 2000; 17 (1): 76-8.

7. García PD, García BC. Eloísa Díaz Insunza y su lucha por la salud escolar en Chile. An Chil Hist Med 2007; 17 (1): 45-51.

8. García PD, García BC. Eloísa Díaz Insunza (1866-1950): Una pionera del feminismo. Rehabil. Integral 2009; 4 (1): 48-51.

9. Egaña Baraona ML, Monsalve Bórquez. Eloísa Díaz y las carencias sociales de los escolares primarios. En: Díaz Insunza, E. Higiene Escolar. Santiago, Chile: Cámara Chilena de la Construcción. Pontificia Universidad Católica de Chile. Biblioteca Nacional; 2011. pág. IX-XLVI.

10. Sepúlveda Carvajal C. Las mujeres chilenas en la medicina. En: Montecino M, compiladora, Mujeres Chilenas: Fragmentos de una historia. Santiago, Chile: Catalonia; 2018. pág. 165-72.

11. Vigarello G. Histoire des pratiques de santé. Le sain et le malsain depuis le Moyen Age. París: Seuil, 1999. (XX) Foucault M. Defender la sociedad. Curso en el College de France (1975-1976). Buenos Aires: FCE, 2000. https://www.uv.mx/tipmal/files/2016/10/M-FOUCAULT-DEFENDER-LA-SOCIEDAD.pdf. 
12. Foucault M. Vigilar y castigar. Nacimiento de la prisión. Buenos Aires: FCE, 1976.

13. Foucault M. Nacimiento de la medicina social. En Foucault M. Obras esenciales II. Madrid, España: Paidós; 1999, pág. 363-84.

14. Miquel J. Catecismo Hijiénico o Arte de Conservar la Salud. Santiago: Del Ferrocarril, 1859.

15. Ibarra M. Higiene y salud urbana en la mirada de médicos, arquitectos y urbanistas durante la primera mitad del Siglo XX en Chile. Rev Med Chile 2016; 144: 116-23.

16. Díaz E. Breves observaciones sobre la aparición de la pubertad en la mujer chilena. Anales de la Universidad de Chile 1887; 71 (1): 893-917. doi: 10.5354/07178883.2012.21739.

17. Ellenberger H. El descubrimiento del inconsciente. Santiago: Gredos, 1976.

18. Morel B. Traité des dégénérescence physiques, intellectuelles, et morales de l'espèce humaine. Paris: Arno, 1857.

19. Díaz E. Reorganización del sistema médico escolar. San- tiago: Imprenta Nacional, 1901.

20. Díaz E. Recopilación de informes del médico-inspector de las escuelas públicas de Santiago: presentadas al Ministerio de Instrucción Pública. Santiago: Imprenta Nacional, 1905.

21. Froebel F. La educación del hombre. Nueva York: Appleton \& Co. 1885.

22. Salas G, Scholten H. Rey-Anacona, C. Nadie nace civilizado: Hacia una crítica del pathos escolar. En Saavedra, E. Salas, G. Cornejo, C. Morales, P. Resilencia y calidad de vida. La psicología educacional en diálogo con otras disciplinas. Talca: Universidad Católica del Maule; 2015, p. 61-73.

23. Hendrick H. Child Welfare: Historical Dimensions, Contemporary Debate. Bristol: Bristol University Press, 2010.

24. Rojas Flores J. Historia de la infancia en el Chile republicano, 1810-2010. Santiago: JUNJI, 2010.

25. Vargas Catalán E. Historia de la pediatría chilena: Crónica de una alegría. Santiago: Editorial Universitaria, 2002. 